\title{
EchoGéo
}

$37 \mid 2016$

Femmes et migrations : celles qui restent

\section{Les migrantes de retour dans la Corne de l'Afrique}

Vers une transformation sociale des espaces émetteurs : le cas éthiopien

Amina Saïd Chiré and Bezunesh Tamru

\section{(2) OpenEdition}

\section{Journals}

Electronic version

URL: https://journals.openedition.org/echogeo/14708

DOI: $10.4000 /$ echogeo. 14708

ISSN: 1963-1197

Publisher

Pôle de recherche pour l'organisation et la diffusion de l'information géographique (CNRS UMR 8586)

Electronic reference

Amina Saïd Chiré and Bezunesh Tamru, "Les migrantes de retour dans la Corne de l'Afrique ", EchoGéo [Online], 37 | 2016, Online since 07 October 2016, connection on 10 August 2021. URL: http:// journals.openedition.org/echogeo/14708 ; DOl: https://doi.org/10.4000/echogeo.14708

This text was automatically generated on 10 August 2021

EchoGéo est mis à disposition selon les termes de la licence Creative Commons Attribution - Pas d'Utilisation Commerciale - Pas de Modification 4.0 International (CC BY-NC-ND) 


\section{Les migrantes de retour dans la Corne de l'Afrique}

Vers une transformation sociale des espaces émetteurs : le cas éthiopien

Amina Saïd Chiré and Bezunesh Tamru

\section{Introduction}

1 La Corne de l'Afrique est une région caractérisée par d'intenses circulations internes et, depuis plusieurs décennies, par des migrations internationales importantes. Notre contribution se place dans ce contexte régional pour interroger plus particulièrement les modes d'installation et de réinsertion socio-spatiales des migrantes de retour volontaire et définitif en Éthiopie. Ce questionnement participe à la réflexion ouverte par ce numéro d'EchoGéo sur la pluralité des formes des «rester». Nous nous intéressons aux mutations insufflées par les migrantes de retour sur l'organisation sociale des espaces émetteurs, ce qui implique de comprendre leurs influences dans les transformations socio-économiques en cours, en particulier dans le secteur de l'immobilier résidentiel en périphérie urbaine. Nous avançons ainsi l'hypothèse que ces femmes ont eu recours à une mobilité pour accéder à une ascension sociale comme les migrants du sexe opposé, mais également pour s'affranchir d'un certain nombre de pesanteurs économiques et sociales qui les maintiennent souvent dans une condition d'infériorité, même si cette décision les expose à une aliénation familiale comme sociale.

2 De retour chez elles, elles se doivent de mettre en œuvre les conditions de réalisation de leur ascension sociale et de jouissance d'une certaine liberté. À ce titre, elles peuvent être considérées comme un des vecteurs des changements sociétaux comme économiques en cours. Car si la période de migration se caractérise par des envois de ressources vers leurs localités d'origine qui connaissent ainsi une certaine aisance économique, à leur retour les migrantes privilégient une mobilité résidentielle considérée comme plus flatteuse. Une réinstallation réussie des migrantes se caractérise ainsi par une nouvelle mobilité localement. 
Une consultation rapide de la littérature scientifique sur les migrations dévoile un faible investissement dans le champ migratoire que forment la Corne de l'Afrique et la Péninsule Arabique, et la relation entre genre et migration est encore moins mobilisée. Notre objectif est également de combler cette lacune par une étude portant sur des migrantes d'origine rurale comme urbaine et qui se sont réinstallées volontairement en Éthiopie.

4 Notre démonstration est construite autour de trois parties. Un examen rapide des migrations introduira la question des déterminants et des implications de la mobilité féminine dans la Corne de l'Afrique. La deuxième partie positionnera la question des migrations féminines au sein de ce contexte régional, avant de caractériser plus longuement les migrantes qui ont choisi de rentrer définitivement en Éthiopie. La dernière partie sera consacrée aux mutations sociales portées en germe par ces retours et qui se sont construites via un certain nombre de «transferts de normes tant économiques que sociales » depuis les périodes d'expatriations.

5 Nous adoptons une démarche analytique en utilisant des données primaires issues de deux enquêtes qualitatives. La première menée à Djibouti-ville auprès de 70 migrantes d'origine éthiopienne et somalienne (août 2014) et la seconde auprès de 25 migrantes de retour en Éthiopie (décembre 2015). L'entretien, conduit sous la forme d'une discussion approfondie, a été la méthode retenue pour le recueil des informations. En Éthiopie, une grande marge a été laissée aux récits de vie.

\section{Éléments sur les mobilités féminines dans la Corne de l'Afrique}

\section{Le contexte régional}

6 Les études contemporaines sur les migrations portent plus volontiers sur le bassin méditerranéen, soutenues par la politique des bailleurs de fonds importants comme l'Union Européenne. Pourtant un bassin migratoire proche ${ }^{1}$, avec un effet de vases communiquant avec la mer Rouge, reste encore peu investi par la recherche si ce n'est sous l'angle du seul déterminisme économique. Les mutations des espaces émetteurs sont encore moins observées alors qu'elles sont une clé de compréhension fondamentale de ces grands mouvements migratoires et des stratégies qui se déploient tant au niveau individuel que collectif. La migration est un phénomène complexe à analyser, car au-delà de sa fonction d'ajustement face à des contraintes sociales, culturelles, économiques et environnementales (Clark et al. 2007 ; Mayda, 2007), elle relève d'une conjonction de logiques aussi bien communautaires qu'individuelles (Péridy, 2010). Elle est également le lieu d'expression de nouvelles cultures et de nouveaux comportements. C'est cette complexité que nous tenterons d'appréhender dans cette section.

\section{Les espaces émetteurs}

7 La République de Djibouti, l'Éthiopie et la Somalie forment un espace ayant connu de nombreux conflits générant une forte insécurité et l'augmentation des migrations. La sous-région est aussi et régulièrement soumise à des aléas climatiques, en particulier des sécheresses. L'ensemble de ces risques, conjugués à la vulnérabilité grandissante 
des sociétés locales, participe au croît des migrations. Cependant, ces phénomènes physiques ne sont pas les seuls à éprouver les sociétés locales. Celles-ci supportent de façon routinière des chocs socio-économiques divers à l'origine d'une paupérisation extrême des ménages. En milieu urbain, les déterminants de la migration régionale sont liés à l'augmentation de la pauvreté, la généralisation du chômage et du sousemploi et la faible rémunération du travail. Cette perspective croisée des déterminants de la mobilité serait incomplète si l'on omettait les aspirations individuelles. Les migrations féminines, tout en s'inscrivant dans une tendance globale, se distinguent en partie par une logique plus personnelle. En effet, des auteurs (Lesclingang, 2004 ; Sakho et al., 2010 ; Oso et a.l, 1997) ont déjà noté une migration féminine visant non seulement une ascension sociale mais aussi une volonté d'indépendance et de refus d'une situation de dominée.

\section{Vers de nouveaux espaces plus attractifs}

8 À l'échelle de la sous-région, l'espace de repli et d'attraction étant Djibouti, d'importants flux de réfugiés, en provenance d'Éthiopie et de Somalie, s'y sont dirigés depuis la fin des années 1970. Les migrants des premières vagues ont été assimilés eu égard à leurs nombreux liens linguistiques et ethniques avec les populations locales, Afars et Somalis. Les suivants, essentiellement des Éthiopiens issus d'autres ethnies et des Somaliens du sud, rencontrèrent plus de difficultés à s'intégrer.

9 Mais à partir de l'année 2000, les destinations changent et concernent quasi exclusivement le Moyen-Orient. Ce changement de polarité a transformé le rôle de Djibouti en faisant de son territoire un lieu de transit privilégié entre la Corne de l'Afrique et la péninsule Arabique, avec l'ouverture de nouvelles destinations aux migrants dont beaucoup sont des femmes.

Entre 2007 et 2012, on a assisté à une augmentation exponentielle du nombre total des nouveaux arrivants au Yémen avec 107500 personnes recensées à la dernière date (voir tableau 1). Cette recrudescence est liée à une augmentation importante des candidats éthiopiens. Selon le HCR, ceux-ci représentent désormais trois migrants sur quatre effectuant la traversée de la mer Rouge. Jusqu'en 2008, les migrants somaliens formaient les trois quarts de toutes les arrivées enregistrées au Yémen. Cette inversion des pourcentages de Somaliens et d'Éthiopiens entre 2008 et 2009 semble coïncider avec la fin de l'engagement des Éthiopiens en Somalie. Les migrants tentent ensuit de gagner l'Arabie Saoudite.

Tableau 1 - Nombre de migrants ayant atteint les côtes yéménites

\begin{tabular}{|l|l|l|l|l|l|l|l|}
\hline Année & 2006 & 2007 & 2008 & 2009 & 2010 & 2011 & 2012 \\
\hline Nombre de migrants & 11000 & 26000 & 50000 & 77000 & 53000 & 103000 & 107500 \\
\hline
\end{tabular}

Source : UNHCR

11 Les Oromos représenteraient $80 \%$ des migrants éthiopiens traversant la mer Rouge depuis le territoire djiboutien. Ils viennent principalement des États régionaux de l'Amhara et de l'Oromia (Mohamed Dabaleh, 2015). 
12 Nos enquêtes sur les migrations dans le bassin de la mer Rouge nous ont permis de mettre en évidence deux grandes catégories de migrantes. La première concerne des jeunes paysannes ou nomades qui sont traditionnellement placées en ville comme domestiques au sein des réseaux de connaissances, familiales ou claniques. Elles empruntent souvent les mêmes trajets terrestres que les hommes mais privilégient les " circuits courts» intra régionaux. Elles sont majoritaires dans le groupe étudié à Djibouti, étude menée pendant leur période d'expatriation. La seconde catégorie de migrantes réinstallées en Éthiopie est composée de citadines ayant une stratégie d'ascension sociale clairement établie (Diop., 2008).

\section{La migrante d'origine rurale : une préférence pour les circuits régionaux}

13 Originaires des États régionaux Amhara, Oromia, Somali et Afar en Éthiopie les migrantes d'origine rurale rencontrées à Djibouti-ville sont relativement jeunes et leur niveau d'étude est faible, voire inexistant. Elles sont souvent issues de familles élargies et se distinguent aussi par une durée courte d'expatriation pour rentrer se marier à un âge "décent». Elles montrent une nette préférence pour des trajets courts, moins coûteux, moins rentables et aussi moins risqués².

En migrant, ces femmes espèrent aider leur famille en diversifiant les sources de revenus. Mais lors des entretiens, les femmes développent aussi un discours où elles revendiquent la recherche d'une autonomie individuelle. Pour cette raison, elles sont souvent plus nombreuses que les hommes à prendre seules la décision de migrer afin de répondre à leurs motivations propres. Une femme économiquement autonome est susceptible de voir sa position sociale et surtout familiale revalorisée lors du retour définitif. Ainsi, plus de la moitié des travailleuses domestiques interrogées à Djibouti ont pris seules la décision d'émigrer et nombre d'entre elles affirment l'avoir pris pour satisfaire leurs propres objectifs.

Pour ces raisons, les Éthiopiennes et les Somaliennes sont de plus en plus nombreuses à se rendre à Djibouti-ville, où elles proposent leurs services sur le marché des employées de maison, en plein essor. Ces migrations s'inscrivent également dans les contextes de sous-emploi, d'une population très nombreuse en Éthiopie ${ }^{3}$ et de guerres civiles en Somalie. L'émigration vers Djibouti s'inscrit surtout dans un réseau d'entraide familial et/ou clanique constitué au fil du temps et dont les ramifications s'étendent de part et d'autre des frontières. Le tiers des personnes rencontrées a été informé des opportunités d'emploi existantes à Djibouti par le biais de relations amicales et pour deux tiers d'entre elles, par leur famille. De la même façon, un quart des migrantes interrogées a été accueilli par des amis ou connaissances et près des deux tiers par des parents ou des membres de leur famille. Le réseau social a également joué un rôle important dans le placement des futures employées de maison. Ainsi, entre quatre et cinq employées sur dix ont trouvé un travail grâce à leur réseau social amical ou familial. Face à la demande continuelle de domestiques et l'existence de solides réseaux sociaux, les flux des travailleuses éthiopiennes et somaliennes se sont donc intensifiés. L'enquête nationale auprès des ménages conduite en 2014 à Djibouti estime leur proportion à $6,7 \%$ des actifs, ce qui place cette activité au $6^{\mathrm{e}}$ rang des secteurs économiques, bien avant le secteur primaire. 
Tableau 2 - Pays d'origine des travailleuses domestiques enquêtées à Djibouti

\begin{tabular}{|l|l|}
\hline Pays d'origine & Nombre \\
\hline Djibouti & 3 \\
\hline Ethiopie & 35 \\
\hline Somalie & 30 \\
\hline Puntland & 1 \\
\hline Yémen & 1 \\
\hline Total & 70 \\
\hline
\end{tabular}

Source : enquête de l'auteur, août 2014.

Dans l'échantillon de 2014, les migrantes rurales ont une volonté claire de retour définitif. Lorsqu'elles arrivent à Djibouti, ces dernières sont contraintes au travail domestique par la nécessité de travailler, mais pas seulement. Elles y sont également poussées par le contexte du marché du travail djiboutien. Sur ce marché, les opportunités qu'elles rencontrent sont très limitées, d'autant plus que les jeunes femmes d'origine éthiopienne et somalienne sont associées au travail domestique dans les représentations qui circulent à Djibouti depuis plusieurs décennies. À partir des années 1960, l'urbanisation massive de la société djiboutienne engendre des changements sociaux importants, dont un éloignement des ménages urbains de leurs cercles familiaux ruraux. Cette nouvelle configuration ne leur permet plus de bénéficier d'une aide domestique d'origine familiale. En outre, il est très mal perçu pour les Djiboutiennes, même de milieu modeste, d'investir ce secteur économique. Travailler pour une famille autre que la sienne est vécu comme un déclassement social très mal considéré. Dès lors, les migrantes d'origine éthiopienne et somalienne sont les seules à pourvoir ce type d'emploi. Cette aliénation et cette absence de perspectives expliquent le turn over important sur le marché du travail domestique. Les trois quarts des migrantes interrogées sont des primo-migrantes.

Si pour la moitié d'entre elles l'objectif premier de la migration est l'aide familiale, beaucoup mettent également en avant les motivations personnelles. Ces réponses ne signifient pas qu'elles refusent de subvenir aux besoins de leurs familles, mais montrent plutôt la volonté de prendre leur avenir en main. Atteindre cet objectif ne représente donc pas qu'un simple enrichissement monétaire pour des jeunes femmes issues des classes rurales pauvres et dominées mais cela permet aussi d'affirmer, voire d'imposer, leur nouvelle personnalité forgée pendant leur période d'expatriation. C'est ce qu'elles déclarent lors des discussions approfondies. En effet, elles ne cherchent pas à se placer en rupture de leur société d'origine, mais plutôt à y importer leur modernité citadine individuelle. 


\section{Migrantes éthiopiennes de retour du Moyen-Orient.}

18 Le retour est une donnée majeure inscrite dans la stratégie des migrantes. En ce qui concerne celles qui choisissent Djibouti, la pénibilité du travail effectué et le manque de perspectives sur place ne leur permettent pas d'envisager une installation définitive. Quant à celles qui se dirigent vers le Moyen-Orient, la politique d'accueil des étrangers dans les États du Golfe est très dissuasive et freine toute velléité d'installation pérenne. S'y ajoute la spécialisation par genre des secteurs d'emploi offerts aux migrants qui décourage la formation de couples sur place. Pour les femmes, les pays du MoyenOrient offrent un grand marché de travaux domestiques ${ }^{4}$ au travers des agences de placement plus accessibles aux citadines. Depuis la fin des années 1990, ce mouvement s'est intensifié et touche actuellement tous les pays de la Corne de l'Afrique et en particulier l'Éthiopie.

\section{Retours et réinstallations}

Pour analyser les modes d'insertion des migrantes urbaines de retour du Moyen-Orient, nous nous fondons sur un ensemble d'entretiens réalisés auprès de 25 anciennes migrantes dans trois villes éthiopiennes : Addis-Abeba, Bishoftou ${ }^{5}$ à $45 \mathrm{~km}$ et Hawassa à $275 \mathrm{~km}$ au sud de la capitale. La taille de cet échantillon interdit toute forme de généralisation mais ouvre une porte de lecture sur les manières de rentrer et de « rester" des migrantes. Le choix des trois villes est dicté par le souci de ne pas privilégier la seule capitale et par les indications de nos informateurs.

Un grand nombre de travaux, parmi les plus récents (Waganesh Zeleke et al., 2015), ont traité la question des retours du Monde Arabe. Ils se focalisent surtout sur les expulsions massives ${ }^{6} \mathrm{~d}^{\prime}$ Arabie Saoudite (Lecadet, 2015) des migrants clandestins, en particulier sous l'angle psychologique. Nous avons interrogé des migrantes parties légalement dans le Monde Arabe et dont le retour et l'installation en Éthiopie ont un caractère clairement définitif. Pourtant même les contacts avec ces dernières se sont avérés délicats, les intéressées étant peu enclines à répondre dans une ambiance urbaine, en Éthiopie, devenue paradoxalement plus "stigmatisante» depuis les expulsions massives. Nous avons dû recourir à des informateurs et à des réseaux de connaissances. La méthode adoptée est une grille d'entretien avec peu de questions et laissant une grande place aux récits. Beaucoup ont préféré des discussions par groupes qui ont enrichi les échanges.

\section{Qui sont celles qui choisissent de rentrer pour rester?}

21 Elles sont toutes citadines d'origine et ont été scolarisées ${ }^{7}$, avec une moyenne d'âge de 34 ans dans notre échantillon. La majorité d'entre elles est issue des classes modestes, bien que certaines déclarent avoir des parents fonctionnaires ou anciens propriétaires terriens. En termes ethniques, elles sont quasiment toutes des amharas chrétiennes, dont une moitié d'orthodoxes et une autre partie de protestantes évangélistes. La surreprésentation des amharas chrétiennes dans notre panel vient du biais introduit par nos informateurs issus de ces groupes. La conversion au protestantisme évangélique s'est souvent faite pendant la période d'expatriation et les adeptes de ces églises montrent une plus grande cohésion de groupe. 
22 À une exception près, toutes les migrantes enquêtées sont célibataires, et un quart d'entre elles ont un enfant à charge, souvent né avant leur départ. Les préjugés raciaux découragent, voire rendent impossibles, les mariages inter-ethniques dans les lieux de migration et les anciennes migrantes développent un discours très moralisateur quant aux relations hommes-femmes durant leur période d'expatriation. Pourtant, et selon une responsable publique, l'existence de pratiques de prostitution des migrantes est avérée. Mais au retour, très peu d'entre elles ${ }^{8}$ osent l'afficher et dans les discussions ce thème est resté tabou. Dans l'ensemble, la question des mariages et du rapport à l'autre sexe demeure un sujet sensible, voire douloureux. Elles sont rentrées muées par la volonté de fonder une famille, mais se trouvent en butte au rejet d'une société prude assimilant leur expérience d'expatriation à des pratiques inavouables. Elles disent toutes nourrir une grande méfiance envers les hommes, car elles craignent d'être la proie d'aigrefins tentés par leurs économies.

\section{Expériences migratoires et préparations du retour}

Pour partir, toutes nos enquêtées ont eu recours à une agence de placement légal. Pour la majorité d'entre elles, la décision fait suite à une information ou un conseil d'amies. Certaines déclarent avoir consulté leur famille ou avoir été aidée par un parent. Le départ est souvent imposé par une grande précarité économique et sociale mais aussi par des situations politiques tendues.

La migration est vécue comme une expérience paradoxale, faite d'épreuves d'adaptation ou de violences physiques. La majorité des migrantes reste évasive sur ces difficultés et préfère les évoquer de façon allusive, voire symbolique (Assaf Dahdah, 2012). Elles sont beaucoup plus prolixes sur les apports de leur expatriation en termes de confiance en soi, de capacité à épargner et à faire des projets. Elles insistent en particulier sur leur participation à des associations d'entraide et à des émissions radiophoniques ${ }^{9}$ communautaires.

L'inscription dans un réseau de solidarité, amicale ou familiale, est ainsi primordiale dans la réussite du retour et surtout $d u$ " rester ». L'idée d'un retour final et rapide est une préoccupation constante et un rêve incantatoire dans l'expérience de nos participantes. Selon nos entretiens, la durée moyenne de leurs différentes expatriations est de 9 ans et les pays où elles ont le plus séjourné sont : le Liban, l'Arabie Saoudite, les Émirats Arabes Unis et le Koweït. Certaines ont vécu dans plusieurs de ces États. Nous avons voulu savoir ce qui les avait finalement décidées au retour définitif. Les réponses sont variées, mais les plus fréquemment invoquées sont la fatigue, une santé déclinante et le désir de vivre enfin pour soi. Si pour 30\% d'entre elles, la décision du retour a été mûrement réfléchie et mise en œuvre, les autres indiquent plus des concours de circonstances les ayant fait «sauter le pas ».

\section{Des réinstallations contrastées}

Dans le panel des anciennes migrantes, 40 \% étaient au chômage au moment de l'enquête, les autres déclarent un emploi stable ou se disent installées à leur compte. Pour réussir leur grand retour et leur installation, elles insistent sur le rôle des réseaux de solidarité. Les premières années d'expatriation sont les plus éprouvantes. Elles disposent de peu car elles remboursent les avances pour les frais d'agence et de 
transports et le reste de leur salaire est envoyé à leur famille. La fin de leur premier contrat $^{10}$ est souvent humiliante car elles doivent retourner chez elle sans économie. À partir de la seconde expérience, une meilleure stratégie d'épargne peut être envisagée, mais n'ayant pas de compte bancaire dans le pays hôte, elles doivent procéder par des envois successifs d'argent à des tierces personnes et cette épargne est souvent dilapidée. Seules celles qui disposent d'un soutien familial solide ou d'un réseau de solidarité amicale soudé ont des chances de récupérer leur épargne.

Dans ce schéma contrasté des réinstallations, trois groupes se dégagent dans le panel étudié, "les vaincues", "les actives" et "les lauréates». Les premières sont au chômage, sans épargne et vivent chez des proches avec un statut minoré. Le second groupe comprend les plus jeunes, elles ont rapidement investi leur épargne dans une formation, coiffure, esthétique et ont une activité salariée. Dans la troisième catégorie se trouvent les plus nanties, celles qui ont méticuleusement préparé le retour et qui possèdent des activités commerciales de plus ou moins grande envergure. Nous les appelons les lauréates, car ce sont celles qui ont atteint «le rêve du retour» le plus abouti. Nous allons analyser ces trois catégories en même temps que le groupe de migrantes d'origine rurale observé à Djibouti dans leurs stratégies de réintégration sociale, d'ascension économique et/ou de mobilités résidentielles.

\section{Les migrantes de retour: quels rôles dans les espaces émetteurs?}

\section{Mutations spatiales et mobilités résidentielles : le triomphe des villes}

Les mutations spatiales sont abordées à partir des transferts sociaux et économiques dont les migrantes sont les vecteurs. Les zones rurales connaissent une sorte de gentrification in situ, mais les migrantes, une fois de de retour, se reconvertissent en petites entrepreneuses et abandonnent très vite la campagne pour la ville la plus proche. La recherche de l'ascension sociale passe ainsi par un nouveau type de mobilité locale qui conduit à un déménagement et une installation d'une partie de la famille en ville. Cette nouvelle mobilité est également imposée par leurs nouvelles activités commerciales qui supposent un bassin de clientèle viable. Les migrantes rencontrées à Djibouti prévoient d'abandonner la campagne pour la ville la plus proche. Dans l'État Régional Somali de la Fédération éthiopienne, les villes de réinstallation souhaitées sont celles de Dire Dawa ${ }^{11}$, de Jidjiga ${ }^{12}$ et de Harar ${ }^{13}$. Dans l'État Régional Amhara d'où est originaire une partie de notre échantillon, c'est Bati la ville de réinstallation la plus visée. D'ailleurs cette ville est emblématique de ces retours définitifs et des mutations sociales et spatiales qui les accompagnent. Elle compte depuis quelques années un quartier que ses habitants ont surnommé « Gardiens », quartier construit par d'anciens travailleurs domestiques à Djibouti ${ }^{14}$. En d'autres termes, l'espace rural bénéficie plus des retombées économiques de la période de migration que de celle des retours triomphants et de l'installation pérenne. Nous pouvons donc considérer que les migrations participent à l'accélération de l'urbanisation qui, dans un mouvement centrifuge, concentre hommes et richesses dans les villes. En Éthiopie, le taux d'urbanisation est passé de 14,7 \% en 1995 à $20 \%$ en 2015 (Banque Mondiale, 2015). 
Cette mutation spatiale majeure explique pourquoi les espaces de départ ne coïncident pas aujourd'hui avec les espaces de retour des migrants.

Les autres types de transferts sociaux sont conditionnés par la pérennité des revenus complémentaires générés par les activités commerciales. L'amélioration du logement familial et de son confort sont ainsi une des conséquences du séjour prolongé des migrantes de retour. L'amélioration du bâti passe par le passage à la construction en dur. Les migrantes, revenues de régions plus riches, investissent aussi dans du mobilier de confort, mais également dans des équipements plus ostentatoires tels que la télévision, le téléphone ou le réfrigérateur. Travailleuses domestiques marginalisées et au bas de l'échelle sociale à Djibouti, au Liban ou en Arabie Saoudite, elles veulent se faire une place reconnue dans leur pays. C'est la dialectique du social et du spatial que l'on retrouve également dans les mutations sociales en cours.

30 Les mutations spatiales observées diffèrent selon les catégories définies précédemment. Ainsi, les "vaincues " revenues sans moyens ont réintégré le foyer familial où elles vivent dans un sentiment plus ou moins objectif de marginalité. Pourtant, elles ont financé l'amélioration des logements familiaux et l'achat d'équipements modernes. Dans l'ensemble les habitats modestes qui composent leur quartier ont ainsi un aspect assez pimpant. Mais malgré ces investissements, toutes ont refusé que leur logement voire leur quartier soient pris en photo. Le groupe des « actives » est composé de jeunes employées des salons de coiffure, ou de vendeuses dans divers secteurs. Ce sont les plus jeunes de notre panel et elles se distinguent par une durée relativement courte de migration. Elles ont réintégré le foyer familial ou habitent en colocation dans la capitale ou les grandes villes régionales. Enfin, la catégorie des «lauréates » est celle des ex-migrantes parvenues à s'établir à leur compte et connaissant une mobilité résidentielle réussie. Dans les entretiens, elles soulignent fortement la force de leur réseau de solidarité devenu un partenariat d'affaires. Elles sont à la tête de plusieurs entreprises : pension, laiterie, salon de coiffure, magasin de vêtements.

31 Si le noyau dur est composé de deux femmes de caractère, plusieurs autres anciennes migrantes participent au réseau. Nous avons ainsi pu discuter avec deux d'entre elles, l'une est propriétaire d'un fast food très actif à Hawasa et l'autre possède un grand magasin de vêtements à Shashamanee ${ }^{15}$. Le réseau a débuté sa stratégie d'épargne et d'investissements pendant le période des migrations. Grâce aux revenus de leurs affaires, certaines de ses membres sont devenues propriétaires de leur logement et sont motorisées. Malgré ces réussites, et à part l'une d'entre elles habitant dans une des nouvelles banlieues de la capitale, toutes les autres « lauréates» vivent dans les villes régionales. 
Illustration 1 - Panneau d'un magasin de vêtements pour homme appartenant à un partenariat d'affaires d'ex-migrantes

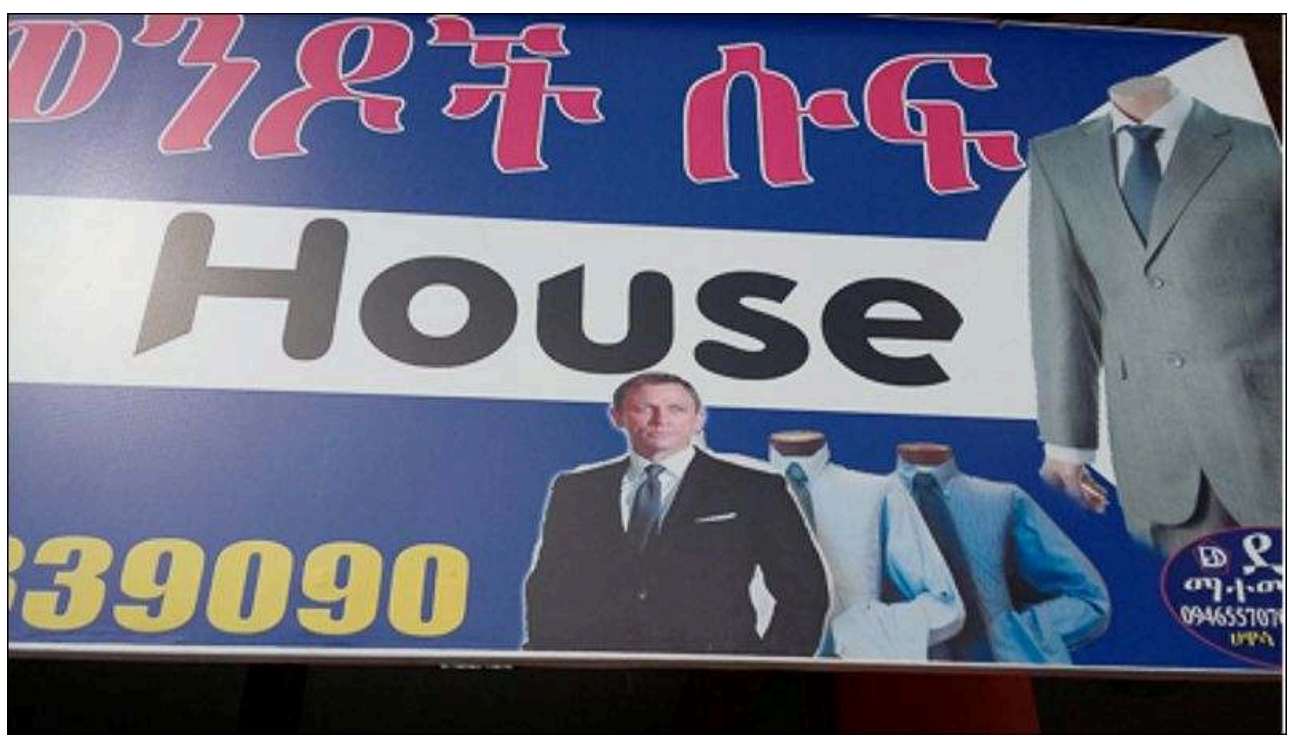

Illustration 2 - Panneau de devanture d'un fast-food portant le nom de Libanose (Liban en amharique), et tenu par une ancienne migrante

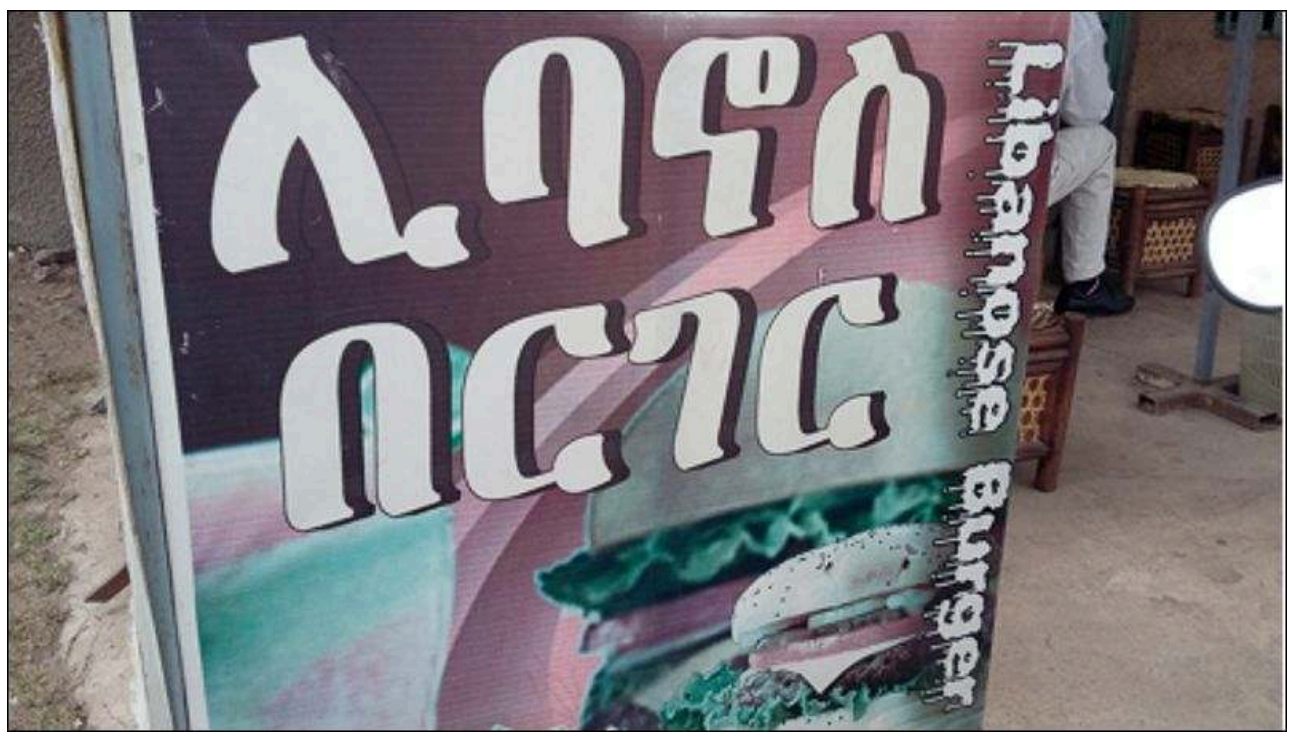

Des intermédiaires immobiliers, interrogés sur ces choix, estiment que les migrantes bénéficiant d'un retour réussi du Moyen-Orient reviennent avec une épargne de 25000 euros en moyenne. Même en investissant la totalité de cette somme, ces dernières ne peuvent donc pas prétendre à un logement décent dans la capitale, si ce n'est dans les quartiers les plus éloignés du centre. Elles ont donc tendance à minimiser leurs prises de risques en investissant dans des petits commerces, des voitures utilitaires ou dans l'achat d'un appartement dans les immeubles collectifs en périphérie lointaine. Ces informations corroborent celles indiquées par les enquêtés sur leurs stratégies de retour. Elles s'installent dans les métropoles régionales comme Hawasa ou des villes secondaires comme Shashamanee où le prix des logements et du foncier urbain, la location de locaux commerciaux, demeurent accessibles et adaptés à l'épargne des migrantes. 


\section{Les mutations sociales} ou masculines. Dans les sociétés de la Corne de l'Afrique, les relations genrées sont basées sur une domination masculine qui trouve ses fondements dans les cultures locales. On peut faire l'hypothèse que les transferts sociaux suite aux migrations féminines sont autant de marqueurs contribuant à brouiller les frontières de classe et de genre traditionnelles. Le retour et la réintégration réussie des anciennes migrantes, devenues salariées, commerçantes voire femmes d'affaires, introduisent de nouvelles normes sociales plus en regard avec le monde extérieur. Même les ex-migrantes restées marginales réinterrogent par leurs expériences les statuts des femmes dans les sociétés locales. Les lignes qui suivent vont permettre de confronter cette hypothèse aux enquêtes et entretiens menés à Djibouti et en Éthiopie.

\section{L'autonomisation des migrantes de retour : quels impacts en milieu rural ?}

volonté d'un retour autonome est clairement affirmée chez les petites bonnes interrogées à Djibouti. C'est d'autant plus étonnant qu'elles sont issues de milieux ruraux considérés comme beaucoup plus fermés. Pourtant, leur retour se réalise dans des conditions de "valorisation" de leur migration qui est acceptée, voire accompagnée, par leur communauté. Situation qui peut s'expliquer par l'extrême pauvreté des mondes ruraux (paysans comme nomades) où l'envoi régulier d'argent par les migrantes répond aux besoins vitaux des familles. C'est aussi grâce à l'épargne ainsi constituée que les jeunes hommes peuvent s'offrir à leur tour une migration plus lointaine (Mohamed Dabaleh, 2015). Dans ces communautés rurales, au demeurant très patriarcales, le rôle de la mère-épouse, en tant que pilier central du foyer, est reconnu et recherché. Les migrantes de retour n'ont donc pas de mal à prouver leur capacité à tenir un foyer et à en diversifier les revenus. Par ailleurs, tout au long de leur séjour à l'étranger en l'occurrence à Djibouti, les proches sont censés connaître et contrôler les lieux de travail des migrantes dans la société d'accueil. Cet encadrement socio-culturel, favorisé par l'existence de réseaux sociaux transfrontaliers, facilite leur réinsertion au retour et leur permet d'envisager un mariage, d'autant plus rapide, que leur épargne les autorise à être moins regardantes sur la dot apportée par le futur époux.

Pourtant beaucoup de ces jeunes femmes veulent, de leur propre chef ou en accord avec leur famille, investir dans une activité commerciale entrainant un déménagement urbain. Mais le statut d'anciennes employées de maison des ex-migrantes est considéré comme dévalorisant dans les sociétés urbaines très stratifiées. L'expérience acquise à l'étranger semble ainsi et paradoxalement mieux admise, voire adoptée, dans les sociétés rurales où les ex-les migrantes jouissent d'une meilleure reconnaissance.

\section{Le retour et la réinstallation des migrantes en ville}

Dans la dernière décennie, l'Éthiopie a connu une croissance économique remarquable allant de 7 à $10 \%$. Celle-ci s'accompagne d'un boom dans l'immobilier, les infrastructures et l'agrobusiness ainsi que d'une industrialisation naissante. Malgré de réelles difficultés dans la vie quotidienne, la société urbaine se reconnait volontiers dans cette nouvelle image du pays loin des représentations de famines des décennies 
précédentes. Mais le pays est aussi devenu un véritable carrefour des migrations clandestines en direction du Moyen-Orient, de la Méditerranée ou de l'Afrique du Sud (Abebaw Minaye, 2015). Les autorités tardent à adopter une politique intégrée sur les migrations et tablent sur leur diminution prochaine grâce à un essor économique décisif. Les anciennes employées de maison de retour du monde arabe, sont ainsi considérées comme une «facette honteuse » d'un développement économique en plein essor et reçoivent un accueil réservé empreint de commisération, voire de rejet.

Durant les entretiens, ce problème est fortement mis en avant. Les ex-migrantes regrettent fortement de voir leur expérience dénigrée par rapport à ceux et celles qui reviennent d'Occident. Le terme de "Diaspora » est réservé à ces derniers, les migrantes du Moyen-Orient étant désignées par le terme dédaigneux d' «Elfora », du nom d'une usine de conditionnement de volailles appartenant à un homme d'affaires éthio-saoudien. Pour pouvoir être acceptées, voire s'imposer dans les sociétés urbaines dont elles étaient souvent issues, les anciennes migrantes doivent donc faire preuve de stratégie. L'adoption d'un discours attendu et auto-culpabilisant fait partie de ces tactiques. En effet, les médias sont remplis de ces témoignages d'anciens migrants, dépeignant à l'envi les horreurs du voyage et du séjour au Moyen-Orient. Le déni de l'expérience moyen-orientale vient surtout des plus jeunes qui comptent ainsi se fondre dans la société urbaine.

C'est la réussite économique, réelle ou supposée, qui prévaut. Lors des entretiens, les anciennes migrantes devenues femmes d'affaires n'ont pas caché leur lassitude face aux fonctionnaires véreux et qui les freinent dans leurs démarches. Les migrantes de retour se sont organisées en réseaux d'amitié et se fréquentent entre elles. Pour les unes, c'est un véritable outil d'ascension sociale et pour les autres, un espace de socialisation et de partage. Dans les discussions de groupes, elles soulignent la méconnaissance de leurs apports par les citadins. En effet, elles revendiquent une expérience forte et solide face à l'adversité, un sens de l'épargne, une aptitude au travail sans compter leurs grandes compétences linguistiques et culinaires. Pourtant, quelles qu'en soient leurs catégories économiques, elles se disent toutes socialement ostracisées et incapables de fonder une famille sur des bases saines.

Ce constat amer issu des entretiens est recoupé par les discours ambiants. Nous avons voulu interroger quelques fonctionnaires célibataires sur le sujet. Les réponses sont tranchées, aucun ne souhaite épouser une femme ayant travaillé au Moyen-Orient. Les raisons du rejet invoquées sont assez ambiguës. Il apparait en creux que ces hommes reprochent surtout aux ex-migrantes d'avoir été au mieux des employées de maison ou au pire des prostituées, et dans les deux cas, d'avoir été soumises à une domination masculine étrangère. Ce dévergondage implicite devient d'autant plus inacceptable que la migration avait été un choix volontaire et revendiqué comme tel par la plupart des femmes et durant plusieurs années. Cette transgression tacite n'est pas plus admise par les citadines qui accusent les migrantes de raréfier l'offre d'employées de maisons et de répandre des mœurs dissolues parmi ces dernières. En effet, le salaire des domestiques à Addis-Abeba a fortement augmenté et beaucoup choisissent la migration moyenorientale plus lucrative tandis que d'autres préfèrent travailler comme manœuvres dans les nombreux chantiers de la ville.

Les migrantes de retour du Moyen-Orient ont ainsi insufflé une mutation sociale marquante dans les villes éthiopiennes, en particulier dans les couches sociales les plus pauvres. Au travers de leurs migrations et leurs retours actifs, elles ont fait preuve 
d'une véritable transgression culturelle et sociale, en faisant bouger les lignes d'une société éthiopienne urbaine patriarcale et très inégalitaire. Celle-ci se "venge » en les cantonnant à un célibat imposé et en les ostracisant par un discours volontairement dévalorisant. Mais les tendances à venir plaident pour elles; en effet ce sont encore des dizaines de milliers de jeunes filles pauvres urbaines et rurales qui se destinent à la migration économique au Moyen-Orient. Elles reviendront de préférence dans les espaces urbains où elles continueront à imposer leurs logiques d'insertion spatiale et leurs normes socio-culturelles forgées pendant des années d'expatriation.

\section{Conclusion}

Le rôle des migrantes dans les mutations spatiales s'exprime par une amélioration continue de l'habitat et des conditions de vie d'innombrables foyers modestes, ruraux comme urbains. Les retours sont de moments de ruptures dans les parcours de vie et doivent donc s'envisager dans leur pluralité. En effet, cet article montre qu'un retour pour être un "rester " réussi et définitif passe par d'innombrables allers/retours internationaux et des mobilités localement renouvelées. Si ces retours et ces "resters » se font plutôt en milieu urbain, le cas éthiopien révèle une nette prédilection pour les installations dans des villes secondaires et les périphéries de la capitale. Cela n'est pas en soi une nouveauté, le rôle des diasporas et leur prise en compte dans l'aménagement des villes ont été souvent soulignés. L'intérêt de nos observations réside dans la façon dont elles réinterrogent ces mutations en identifiant des spécificités tout autant sociales que " genrées ».

Les pays des « retours ", en particulier l'Éthiopie, restent encore frileux dans la prise en compte spécifique de la migration de retour par genre. Certes, les représentations consulaires au Moyen-Orient proposent une inscription dans des projets de construction d'habitats collectifs. Malgré leur enthousiasme, les migrantes ont dû vite déchanter face à la longueur des périodes de versements préalables, peu compatibles avec la précarité de leur mode de vie. Elles préfèrent ainsi investir dans leurs propres réseaux de solidarité, qui restent encore leur moyen d'épargne le plus sûr pour construire un retour réussi. L'augmentation constante du nombre de migrantes, et de façon concomitante de leurs retours, continueront à influencer les espaces émetteurs en y imprimant des mutations sociales spécifiques en raison des rôles de plus en plus majeurs qu'elles y tiennent.

\section{BIBLIOGRAPHY}

Abebaw Menaye (ed.), 2015. Managing the Socio-cultural, Health, Legal and Economic Dimensions of Migration: Emphasis on Ethiopian Migrants in the Middle East and South Africa. Research report, AddisAbeba University, $130 \mathrm{p}$.

Assaf Dahdah, 2012. "L'art du faible" Les migrantes non arabes dans le Grand Beyrouth (Liban). Collection Cahiers de l'Ifpo, Presse de l'Ifpo, Beyrouth, 170 p. 
Clark X., Hatton J., Williamson J., 2007. Explaining us immigration, 1971-1998. Review of Economics and Statistics, vol. 2, 89, p. 359-373.

Boyle P., 2002. Population Geography: Transnational Women on the Move. Progress in Human Geography, $n^{\circ} 26$ (4), p. 531-543.

De Regt M., 2012. Ethiopian Women's Migration to Yemen. Chroniques yéménites, n 17, http:// cy.revues.org/1853

De Regt M., Destremau Bl., Latte Abdallah S., 2012. Genre et mobilités au Yémen et dans la Corne de l'Afrique. Chroniques yéménites, ${ }^{\circ} 17$, http://cy.revues.org/1862

Destremau Bl., 2002. L'émergence d'un marché du travail domestique au Yémen : une étude sur Sana'a. Revue Tiers Monde, XLIII, n 170, p. 327-351.

Diop M.-C., 2008. Le Sénégal des migrations. Paris, Crepos-Karthala-ONU Habitat, 431 p.

Fernandez B., 2010. Cheap and Disposable? The Impact of the Global Economic Crisis on the Migration of Ethiopian Women Domestic Workers. Gender \& Development, n¹8 (2), p. 249-262

Lecadet C, 2015. The expulsion of Ethiopian workers from Saudi Arabia (2013-2014): the management of a humanitarian and political crisis. International Conference - Migration and Exile in the Horn of Africa - state of the knowledge and current debates, Khartoum 17-18 november 2015CEDEG.

Lesclingang M., 2004. Nouvelles stratégies migratoires des jeunes femmes rurales au Mali : de la valorisation individuelle à une reconnaissance sociale. Sociétés Contemporaines, 55, p. 21-42

Mayda A. 2007. International Migration: A Panel Data Analysis of the Determinants of Bilateral Flows. Cepr Discussion Paper, 6289.

Mohamed Dabaleh H., 2015. Le rôle de l'espace djiboutien dans les migrations internationales entre la Corne de l'Afrique et la péninsule Arabique, transits et trafics de migrants irréguliers. Mémoire de Master 1, Université de Paris 8, 78 p.

Oso L., Catarino C., 1997. Femmes chefs de ménage et migration. In Bisilliat J., Femmes du sud et chefs de famille, Paris, Karthala.

Péridy N., 2010. Un modèle généralisé des déterminants des migrations internationales. Application aux migrations des pays méditerranéens vers l'UE. Revue économique, vol. 61, 6 .

Sakho B., Dial F. B., Ndiome B., 2010. Migration clandestine féminine. Étude de cas de Dakar et sa banlieue. CARIM, Notes d'analyse et de synthèse, $\mathrm{n}^{\circ} 56$.

Thiollet H., 2004. Aux marges du monde arabe. Chroniques yéménites, n 12, http://cy.revues.org/ 190

Waganesh Zeleke, Abebaw Minaye, Gibbs Kygana, 2015. Mental health and somatic distress among Ethiopian migrant returnees from the Middle-Eat. International Journal of Mental Health and Psychiatry, vol. 1, 2, p. 1-6.

\section{NOTES}

1. Entre janvier et novembre 2014, 207000 migrants ont traversé la Méditerranée. Pour la première fois cette année-là, les personnes originaires de la Corne de l'Afrique sont devenues une composante essentielle de ce flux. Avec les Syriens, les Érythréens ont ainsi représenté $50 \%$ du total des migrants. La mer Rouge est donc un autre itinéraire maritime important. En 2014, 
82680 personnes l'ont emprunté, en provenance principalement d'Éthiopie et de Somalie et à destination du Yémen ou de l'Arabie saoudite et des pays du Golfe persique (HCR, 2014).

2. Nous avons cependant rencontré quelques migrantes se préparant à se rendre illégalement dans le Golfe arabo-persique, connaissant et acceptant les risques d'un tel voyage, en particulier le viol. Elles nous ont déclaré s'être fait poser des implants contraceptifs.

3. La population totale éthiopienne est estimée à 96,5 millions d'habitants (PNUD, 2015).

4. Les chiffres officiels du Ministère des Finances Éthiopien indiquent la présence en 2013 de 394393 migrantes vivant légalement en Arabie Saoudite, au Koweït et dans les Émirats. Ces chiffres ne tiennent pas compte des migrantes vivant au Liban, estimées à plus de 100000 .

5. Cette ville est aussi désignée par son ancien nom Debre-Zeit.

6. Nous avons consulté sur ce sujet un grand nombre de mémoires du département d'étude en Travaux Sociaux à l'Université d'Addis-Abeba.

7. $30 \%$ ont achevé le niveau primaire et $50 \%$ celui du secondaire tandis que $20 \%$ avait commencé des études supérieures.

8. À Djibouti, ville-garnison internationale, la prostitution des migrantes éthiopiennes s'affiche et les patronnes de boîtes de nuit parviennent même à se forger une certaine respectabilité sur place et dans leurs régions d'origine. Mais au Moyen-Orient, cette même pratique est cachée, voire dangereuse selon les pays, et s'adresse à une clientèle d'immigrés pauvres.

9. Au Liban, des associations caritatives catholiques, très actives auprès des migrants, mettent à la disposition des plages horaires d'ondes radiophoniques.

10. Les migrantes légales sont sous contrat et leur permis de séjour est lié à la durée du contrat aussi doivent-elles repartir chez elles pour en attendre le renouvellement.

11. Seconde ville éthiopienne avec administration autonome et 600000 habitants (2008).

12. Capitale de l'Etat Régional Somali en Éthiopie, Jigjiga compte 100000 habitants.

13. 127000 habitants (est. 2007).

14. Gardiens pour les hommes et employées de maison pour les femmes.

15. Ville située à $250 \mathrm{~km}$ au sud de la capitale avec 100454 habitants (2007).

\section{ABSTRACTS}

The growing demand for maids in the Republic of Djibouti and in the Middle East countries encourage female migration from Ethiopia and from Somalia. During their stays abroad, migrant women will have several strategies in order to return successfully back home. Our study thus questions the ways these return are implemented in term of resettlements and socio-spatial reintegration in Ethiopia. Indeed, the study shows that a "return" to be a final and rewarding "stay" must undergo countless international trips followed by several domestic mobilities. These returns and "final staying's" are more frequent in urban areas, the Ethiopian case shows also that secondary towns and the suburbs of the capital city are areas mostly preferred by returnees. Beyond these facts, the findings of the study unveil the way female returnees readdress the question of urban changes by their social as well as by their gender specificities.

La demande croissante en personnel domestique dans la République de Djibouti et dans les pays $\mathrm{du}$ Moyen-Orient encourage la migration de femmes originaires d'Éthiopie et de la Somalie. Pendant leurs périodes d'expatriation, les migrantes mettent en œuvre plusieurs stratégies pour réussir un retour synonyme d'ascension sociale. Notre étude interroge ces retours en termes de 
modes d'installation et de réinsertion socio-spatiales en Éthiopie. En effet, l'étude montre qu'un «retour" pour être un " rester» abouti et définitif doit passer par d'innombrables allers/ retours internationaux suivis par des mobilités localement renouvelées. Les retours et les «resters » privilégient le milieu urbain, le cas éthiopien révèle ainsi une nette prédilection pour les installations dans des villes secondaires et les périphéries de la capitale. Au-delà, nos observations dévoilent la façon dont les migrantes de retour réinterrogent les mutations urbaines par leurs spécificités tout autant sociales que « genrées ».

\section{INDEX}

Mots-clés: Corne de l'Afrique, migrante, retour, mutation sociale, Éthiopie, mobilité résidentielle

Keywords: Horn of Africa, female migrant, return, social change, Ethiopia, residential mobility Subjects: Sur le Champ - Sur le Terrain

\section{AUTHORS}

\section{AMINA SAIID CHIRÉ}

Amina Saïd Chiré, amina.said@hotmail.fr, est Maître de conférences à l'Université de Djibouti et membre de l'UMR LADYSS. Elle a publié récemment :

- Said Chiré A. (dir), 2012. Djibouti contemporain. Paris, Karthala, 360 p.

- Said Chiré A., 2015. De la production sociale de la ville à la production de vulnérabilités, l'exemple de la ville de Djibouti. Territoire en mouvement. Revue de géographie et aménagement, [En ligne], 27-28 | 2015, mis en ligne le 06 novembre 2015, http://tem.revues.org/3157

- Saïd Chiré A., 2016. Réseaux et territoires de l'islam politique en République de Djibouti. Territoires d'Afrique, Les territoires en crise violente en Afrique, 8.

\section{BEZUNESH TAMRU}

Bezunesh Tamru, bezunesh.tamru@univ-paris8.fr, est Professeure à l'Université Paris 8 et membre de l'UMR LADYSS. Elle a publié récemment :

- Tamru B., 2011. Ethiopian rural facilities and services. Direction d'une série d'atlas régionaux en onze volumes, Central Statistical Agency (CSA) - UNFPA.

- Tamru, B., 2012. Le rôle du port de Djibouti dans la construction urbaine et contemporaine du territoire éthiopien : vers un arc économique et maritime dans la Corne de l'Afrique. In Amina Said Chiré (dir.), Djibouti contemporain. Université de Djibouti, Karthala, p. 159-175.

- Tamru B., 2013. Villes et Territoires en Éthiopie. L'Harmattan, 439 p. 